\title{
The Ras-ERK signaling pathway regulates acetylated activating transcription factor 2 via p300 in pancreatic cancer cells
}

\author{
Mu Li ${ }^{1}$, Shao-Wei Song ${ }^{2}$, Yang Ge ${ }^{1}$, Jun-Yi Jin ${ }^{1}$, Xiao-Ying Li $^{2}$, Xiao-Dong Tan ${ }^{1}$ \\ ${ }^{1}$ Department of General Surgery, Shengjing Hospital of China Medical University, Shenyang, China; ${ }^{2}$ Department of General Surgery, the First \\ Affiliated Hospital of China Medical University, Shenyang, China \\ Contributions: (I) Conception and design: M Li, XD Tan; (II) Administrative support: Y Ge; (III) Provision of study materials or patients: M Li, JY Jin, \\ XY Li; (IV) Collection and assembly of data: SW Song; (V) Data analysis and interpretation: M Li, XD Tan; (VI) Manuscript writing: All authors; (VII) \\ Final approval of manuscript: All authors. \\ Correspondence to: Dr. Xiao-Dong Tan. Department of General Surgery, Shengjing Hospital of China Medical University, No. 36 Sanhao Street, \\ Heping District, Shenyang, China. Email: tanxdcmu@163.com.
}

Background: Activating transcription factor 2 (ATF2) regulates the expression of downstream target genes and is phosphorylated by the Ras-extracellular-signal-regulated kinase (ERK) pathway. Acetylation of ATF2 is necessary for this type of regulation. However, the molecular mechanism by which the Ras-ERK pathway mediates the regulation of acetylated ATF2 is unknown. This study investigates the mechanism of Ras-ERK pathway-mediated regulation of acetylated ATF2 in maintaining the characteristic phenotype of pancreatic cancer cells.

Methods: This study was carried out using ASPC-1 and BXPC-3 pancreatic cancer cell lines transfected with the double mutant Ras ${ }^{\mathrm{G} 12 \mathrm{~V} / \mathrm{T} 35 \mathrm{~S}}$. The levels of phosphorylated ERK1/2 were measured to establish the activated Ras-ERK pathway. The regulation of acetylated ATF2 was examined by detecting the protein level using western blotting, and the effects on cancer cell phenotype were measured using cell viability, proliferation, migration, and apoptosis assays. Also, chromatin immunoprecipitation (ChIP) assays were used to measure the effect on respective downstream target genes.

Results: The results showed that Ras ${ }^{\mathrm{G} 12 \mathrm{~V} / \mathrm{T} 35 \mathrm{~S}}$ reduced the level of acetylated ATF2 in ASPC-1 and BXPC-3 cells. Compared to wild-type ATF2, the mutant ATF2 ${ }^{\mathrm{K} 357 \mathrm{Q}}$ (which mimics the irreversible acetylated form of ATF2) reduced the cancer cell phenotype and showed decreased enrichment on target genes upon transfection with Ras. Moreover, the level of acetylated ATF2 was regulated by the degradation of p300 through E3 ubiquitin ligase mouse double minute 2 homolog (MDM2).

Conclusions: Activation of the Ras-ERK pathway regulates acetylated ATF2 through degradation of p300 via a proteasome-dependent pathway, which alters the transcription of downstream target genes responsible for the cancer cell phenotype.

Keywords: Ras-extracellular-signal-regulated kinase (ERK) pathway; activating transcription factor 2 (ATF2) acetylation; ASPC-1 cell; BXPC-3 cell; histone deacetylase 2 (HDAC2); P300

Submitted Jul 29, 2020. Accepted for publication Sep 25, 2020.

doi: $10.21037 / \mathrm{atm}-20-5880$

View this article at: http://dx.doi.org/10.21037/atm-20-5880

\section{Introduction}

Pancreatic cancer is among the most common cancers and presents a major challenge for clinicians due to its asymptomatic nature. Typically, diagnosis is only possible in advanced stages of the disease and this reduces survival rates considerably. Almost $95 \%$ of pancreatic cancers are exocrine in nature, and among these, the most common is pancreatic ductal adenocarcinoma (PDAC) (1-3). As for treatment, surgery is the only potentially curative treatment for pancreatic adenocarcinoma; unfortunately, at most only 
$20 \%$ of newly diagnosed patients have disease suitable for curative resection after careful pretherapeutic staging (4). After curative resection for pancreatic cancer, $69-75 \%$ of patients relapse within 2 years and $80-90 \%$ within 5 years $(5,6)$. This high rate of disease recurrence following surgery provides a strong rationale for the need for adjuvant therapy and chemoradiotherapy (CRT) to improve overall survival following resection (7). Gemcitabine, a standard care cytotoxic nucleoside analog, is used very scarcely and achieves a median survival time of only 5 months $(8,9)$. Treatment with a combination of chemotherapeutic agents and targeting of specific molecular pathways can significantly improve the outcomes. In this regard, the extracellular signal-regulated protein kinases (ERK) 1 and 2 are currently being explored $(9,10)$.

The Ras-ERK signaling pathway, which controls cell proliferation, survival, differentiation, and motility, is often found to be upregulated in pancreatic cancer (11). Although the mechanism of epigenetic regulation is not fully understood, it is known that the mutations or growth factors activate the three-tiered Ras-ERK signaling pathway. In this case, Ras guanosine triphosphatase (GTPase) acts upstream of MEK (mitogen-activated protein kinase), which is upstream of ERK (12-15). In the ductal adenocarcinoma model, inhibition of the Ras-ERK pathway blocks cellular proliferation and limits metastasis. However, in the development of novel therapeutics, targeting of downstream kinases is preferable to oncogenic RAS (16).

Activating transcription factor-2 [ATF2, also known as cAMP response element-binding protein 1 (CRE$\mathrm{BP} 1)]$ is a helix-loop-helix transcription factor that has been associated with a various cancers due to its role in maintaining the cancer cell phenotype (17-20). Physically distinct, functional domains of ATF2 impart sequencespecific deoxyribonucleic acid (DNA) binding and regulate transcriptional functions (21). The Ras signal cascade phosphorylates ATF2 to promote carcinogenesis by altering the transcription of downstream genes. However, a dominant-negative ATF2 could block cancer cell proliferation (22). P300 is one of global transcriptional coactivators that turns tightly wrapped around a central histone octamer is involved in the regulation of various DNA-binding transcriptional factors (23). P300 and CREB-binding protein (CBP), as global transcriptional coactivators are capable of histone acetyltransferase activity (24). It is known that p300 performs acetylation of ATF2 at the Lys ${ }^{357}$ position (25), which is important for the downstream histone modification at the target binding sites. However, its role in pancreatic cancer is still unknown.

In this study, we report that the Ras-ERK signaling pathway regulates acetylated ATF2 (ATF2 ${ }^{\mathrm{K} 357 \mathrm{ac}}$ ) by inducing proteasome-dependent degradation of the $\mathrm{p} 300$ protein. Interestingly, we found that p300 was degraded only at the protein level without affecting its transcription status. This in turn affects the expression of downstream genes that are involved in maintaining the pancreatic cancer cell phenotype. Overall, our results suggest a potential mechanism for the regulation of $\mathrm{ATF} 2^{\mathrm{K} 357 \mathrm{ac}}$ in the progression of pancreatic cancer.

We present the following article in accordance with the MDAR reporting checklist (available at http://dx.doi. org/10.21037/atm-20-5880).

\section{Methods}

\section{Cells culture and transfection}

ASPC-1 and BXPC-3 pancreatic cancer cell lines were obtained from the American Type Culture Collection (ATCC, Manassas, USA). The cells were maintained at $37{ }^{\circ} \mathrm{C}$ with $5 \%$ carbon dioxide $\left(\mathrm{CO}_{2}\right)$ in Dulbecco's Modified Eagle Medium (DMEM) with 10\% fetal bovine serum (FBS), $100 \mu \mathrm{g} / \mathrm{mL}$ streptomycin, and 100 units $/ \mathrm{mL}$ penicillin. These cells were grown overnight to achieve transfection of the respective plasmids and small interfering ribonucleic acids (siRNAs) using lipofectamine 3000 (Invitrogen) according to the manufacturer's instructions. The cells were utilized for the experiments after $48 \mathrm{~h}$ of transfection.

\section{Plasmids and siRNA}

Plasmids were generated for the respective coding regions of human ATF2, p300, histone deacetylase 2 (HDAC2), mouse double minute 2 homolog (MDM2), and Ras [wildtype Kirsten (K)] from the pancreatic cancer cells (ASPC1 and BXPC-3) using polymerase chain reaction (PCR) amplification. All plasmids were confirmed by sequencing. Mutant enhanced green fluorescent protein (pEGFP)$\mathrm{Ras}^{\mathrm{G} 12 \mathrm{~V}}$ was obtained using site-directed mutagenesis, and the resultant template (pEGFP-Ras ${ }^{\mathrm{G} 12 \mathrm{~V}}$ ) was used to construct the double mutant pEGFP-Ras ${ }^{\mathrm{G} 12 \mathrm{~V} / T 35 \mathrm{~S}}$. HDAC2 (Shanghai GenePharma), ATF2, and ATF2 ${ }^{\mathrm{K} 357 \mathrm{Q}}$ were also generated (TaKaRa Mutant BEST Kit). 


\section{$R N A$ extraction and quantitative real-time polymerase chain reaction ( $q R T-P C R)$}

For ribonucleic acid (RNA) isolation, cell lysis was performed in the tissue culture plates using TRIzol reagent (Invitrogen), and subsequently subjected to deoxyribonuclease I (DNase-I) treatment. Using the respective oligo-deoxythymine (dT) primers (Invitrogen) and Moloney Murine Leukemia Virus (M-MuLV) reverse transcriptase (Fermentas), synthesis of the first-strand complementary DNA (cDNA) was conducted from the total RNA. Real-time PCR was performed using a QuantiTect SYBR Green PCR Kit (Qiagen). Glyceraldehyde 3-phosphate dehydrogenase (GAPDH) was used as an internal control. All samples were analyzed in triplicate according to the manufacturer's instructions. The respective primer sequences are provided in the supplementary data (Table S1).

\section{Western blot analysis}

The cells were washed in phosphate-buffered saline (PBS) and lysis was carried out in the radioimmunoprecipitation assay (RIPA) buffer on ice. Lysates were centrifuged to obtain the supernatants and the total protein concentrations were determined. These were then transferred to the nitrocellulose membranes, followed by incubation with anti-ATF2 antibody (1:500), anti-ERK1/2 antibody (1:500), anti-phospho-ERK1/2 ${ }^{\mathrm{T} 202}$ antibody (1:500), anti-HDAC2 antibody (1:500), anti-hemagglutinin (HA) antibody (1:500), anti-green fluorescent protein (GFP) antibody (1:500), anti-histone (His) H3 antibody (1:500), anti-p300 antibody (1:500), anti-MDM2 antibody (1:500), and anti- $\beta$-actin antibody $(1: 1,000)$ at $4^{\circ} \mathrm{C}$ overnight. All antibodies were purchased from Abcam (Abcam, Cambridge, UK). After this, incubation with secondary horseradish peroxidase (HRP)-conjugated anti-mouse antibodies was conducted for $1 \mathrm{~h}$. Protein bands were illuminated using the luminal agent SC-2048 (Santa Cruz Biotechnology, California, USA) according to the manufacturer's instructions.

\section{Cell viability [cell counting Kit-8 (CCK-8)] assay}

The cells were seeded and incubated for $24,48,72$, and $96 \mathrm{~h}$ in $96-$ well culture plates. These were mixed and incubated with CCK-8 (Thermo Fisher Scientific, USA) for another $3.5 \mathrm{~h}$ to assess the transfected cells $\left(5 \times 10^{3}\right.$ cells/well). The absorbance of each well was measured at $450 \mathrm{~nm}$ using an Emax spectrophotometer (Thermo Fisher Scientific).

\section{Cell proliferation (colony formation) assay}

For the soft-agar colony formation assay, the transfected cells were suspended in DMEM containing $0.35 \%$ lowmelting agarose. These were then plated onto solidified $0.6 \%$ agarose in DMEM in six-well culture plates at a density of $1 \times 10^{3}$ cells per well. After 3 weeks of incubation, the number of colonies was observed microscopically.

\section{Transwell (cell migration) assay}

The cell migration was measured using the transwell migration assay. $200 \mu \mathrm{L}$ suspensions of transfected cells $\left(1 \times 10^{4}\right.$ cells/well) in serum-free medium were added to the upper chambers and $600 \mu \mathrm{L}$ DMEM containing $10 \%$ FBS was added into the lower chambers. After $48 \mathrm{~h}$ of incubation, migrated cells were fixed with methanol and stained with crystal violet. Finally, the stained cells were counted using a light microscope (Olympus Corporation, Tokyo, Japan).

\section{Flow cytometry and cell apoptosis}

Cell apoptosis was detected using the Annexin V-FITC kit (Biosea Biotechnology Co., Beijing, China). The transfected cells, resuspended in PBS buffer $\left(5.0 \times 10^{5}\right.$ cells $\left./ \mathrm{mL}\right)$, were double-stained with Annexin V-Alexa Fluor 647 and propidium iodide (PI). The apoptotic rate was measured using a flow cytometer (BD Biosciences, USA).

\section{Cbromatin immunoprecipitation assay (ChIP)}

Sample $\left(3 \times 10^{6}\right.$ transfected cells/sample $)$ cross-linking was carried out in $1 \%$ formaldehyde for $10 \mathrm{~min}$ at room temperature (RT). Samples were washed twice with ice cold PBS prior to lysis in sodium dodecyl sulfate (SDS) Lysis Buffer (Upstate, \# 20-163). Lysates were then sonicated (Ultrasonic bath, Bioruptor, Diagenode) to shear the DNA to an average length of 200-800 base pairs (bp). These were then centrifuged at $15000 \mathrm{~g}$ to collect the supernatants, followed by dilution in ChIP Dilution Buffer (Upstate, \#20-153). Immunoprecipitation was carried out overnight with $2 \mu \mathrm{g}$ rabbit anti-ATF2 ${ }^{\mathrm{K} 357 \mathrm{ac}}$ antibody (synthesized by Cell Signaling Technology, Danvers, Massachusetts, USA) at $4{ }^{\circ} \mathrm{C}$. For the control, immunoprecipitations were performed using $2 \mu \mathrm{g}$ normal anti-immuoglobulin 

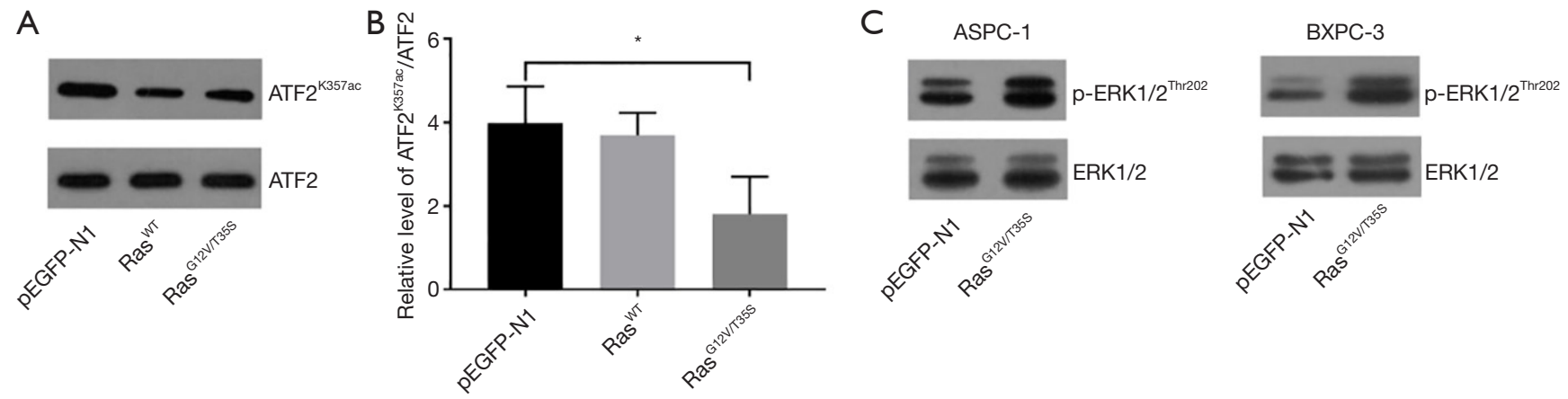

Figure $1 \mathrm{ATF}^{\mathrm{K} 357 \mathrm{ac}}$ is regulated by the ERK1/2 pathway. The ATF2 ${ }^{\mathrm{K} 357 \mathrm{7c}}$ levels in empty-pEGFP-N1 (vector), pEGFP-Ras ${ }^{\mathrm{WT}}$, pEGFPK-Ras ${ }^{\text {G12V/T35S }}$ plasmids were measured using (A) Western blotting and (B) quantitative estimation. (C) Western blots for the levels of p-ERK1/2 ${ }^{\text {Thro2 } 22}$ and ERK1/2 in Ras ${ }^{\text {G12V/T35s }}$ and pEGFP transfected cells (ASPC-1 and BXPC-3). *, P<0.05 vs. pEGFP-N1.

G (IgG) antibody. The beads were subjected to three sequential washes $\left(5 \mathrm{~min}\right.$ each at $\left.4{ }^{\circ} \mathrm{C}\right)$ in the following buffers: low-salt (Upstate, \#20-154), high-salt (Upstate, \#20-155), and lithium chloride (LiCl) (Upstate, \#20-156). Lastly, two further washing steps were performed with Tris- Ethylenediaminetetraacetic acid (TE) (1×; Upstate, \#20-157) for 2 min at RT, and DNA was eluted in 1\% SDS/100 $\mathrm{mM}$ sodium bicarbonate $\left(\mathrm{NaHCO}_{3}\right)$ for $15 \mathrm{~min}$ at RT. Crosslinking was reversed in $200 \mathrm{mM}$ sodium chloride $(\mathrm{NaCl})$ for $7 \mathrm{~h}$ at $65^{\circ} \mathrm{C}$. The eluted DNA was precipitated with ethanol at $-20{ }^{\circ} \mathrm{C}$ overnight. Samples were analyzed by quantitative real-time polymerase chain reaction (qRTPCR) using $1.5 \mu \mathrm{L}$ immunoprecipitated DNA and the serial dilutions of $10 \%$ input DNA (1:4, 1:20, 1:100, and 1:500).

\section{Statistical analysis}

Data analysis was carried out using SPSS 20.2 (SPSS, Chicago, IL, USA) and GraphPad Prism 7 (La Jolla, CA, USA) software, and Student's (two-tailed) $t$-test or a oneway analysis of variance (ANOVA) was applied. Data were presented as mean \pm standard deviation $(\mathrm{SD})$. A minimum of three experiments were completed independently. $\mathrm{P}<0.05$ was considered statistically significant.

\section{Results}

\section{Acetylated ATF2 is regulated by the Ras-ERK pathway}

Western blot analysis of the transfected cells showed that compared to the control (empty vector, pEGFP-N1) or Ras-wild-type (WT) (pEGFP-Ras ${ }^{\mathrm{WT}}$ ), transfection of the double mutant K-Ras ${ }^{\mathrm{G} 12 \mathrm{~V} / \mathrm{T} 35 \mathrm{~S}}$ (pEGFP-Ras ${ }^{\mathrm{G} 12 \mathrm{~V} / \mathrm{T} 35 \mathrm{~S}}$ ) significantly downregulated the level of acetylated ATF2 protein $\left(\mathrm{ATF}^{\mathrm{K} 357 \mathrm{ac}}\right)(\mathrm{P}<0.05)$ (Figure $\left.1 A, B\right)$. Also, when the activation of mitogen-activated protein kinase (MAPK) was measured by detecting the level of phosphorylated ERK1/2 (p-ERK1/2 ${ }^{\text {Thr202 }}$ ), we found that activation was only evident in the Ras ${ }^{\mathrm{G} 12 \mathrm{~V} / \mathrm{T} 35 \mathrm{~S}}$-expressing cells, indicating the activation of the downstream pathway. Here, total ERK1/2 proteins were used as loading controls (Figure 1C). Overall, these results indicate that acetylated ATF2 is regulated by the Ras-ERK pathway.

\section{Mutant ATF $2^{K 357 Q}$ inbibits cell proliferation and migration mediated by Ras-ERK patbway}

A lysine-to-glutamine $(\mathrm{K} \rightarrow \mathrm{Q})$ substitution is a known acetyl mimic that has been used to study the effect of acetyl modification in many acetyltransferases. It is a stable modification that inhibits deacetylation. In this study, we created an $\mathrm{ATF} 2^{\mathrm{K} 357 \mathrm{Q}}$ mutant to mimic the acetylated form of ATF2. To measure the effects of acetylated ATF2 on cell viability, colony formation, migration, and apoptosis, we used CCK-8, soft-agar, transwell migration, and flow cytometry assays, respectively. The results from these assays demonstrated that expression of Ras and ATF2 increased cell viability (Figure 2A), cell numbers (Figure 2B), and cell migration (Figure 2C). Although, cell apotosis decreased $(\mathrm{P}<0.01)$ (Figure $2 D)$ in the ASPC-1 and BXPC cells. However, the transfection of ATF2 ${ }^{\mathrm{K} 357 \mathrm{Q}}$ (acetylated, inactive ATF2) in place of ATF2 restored the cell phenotype (Figure $2 A, B, C, D)$. An empty vector (GFP) along with ATF2 was used as a control. These results strongly indicate that the acetylation of ATF2 is regulated by the Ras-ERK pathway in pancreatic cancer cells. 

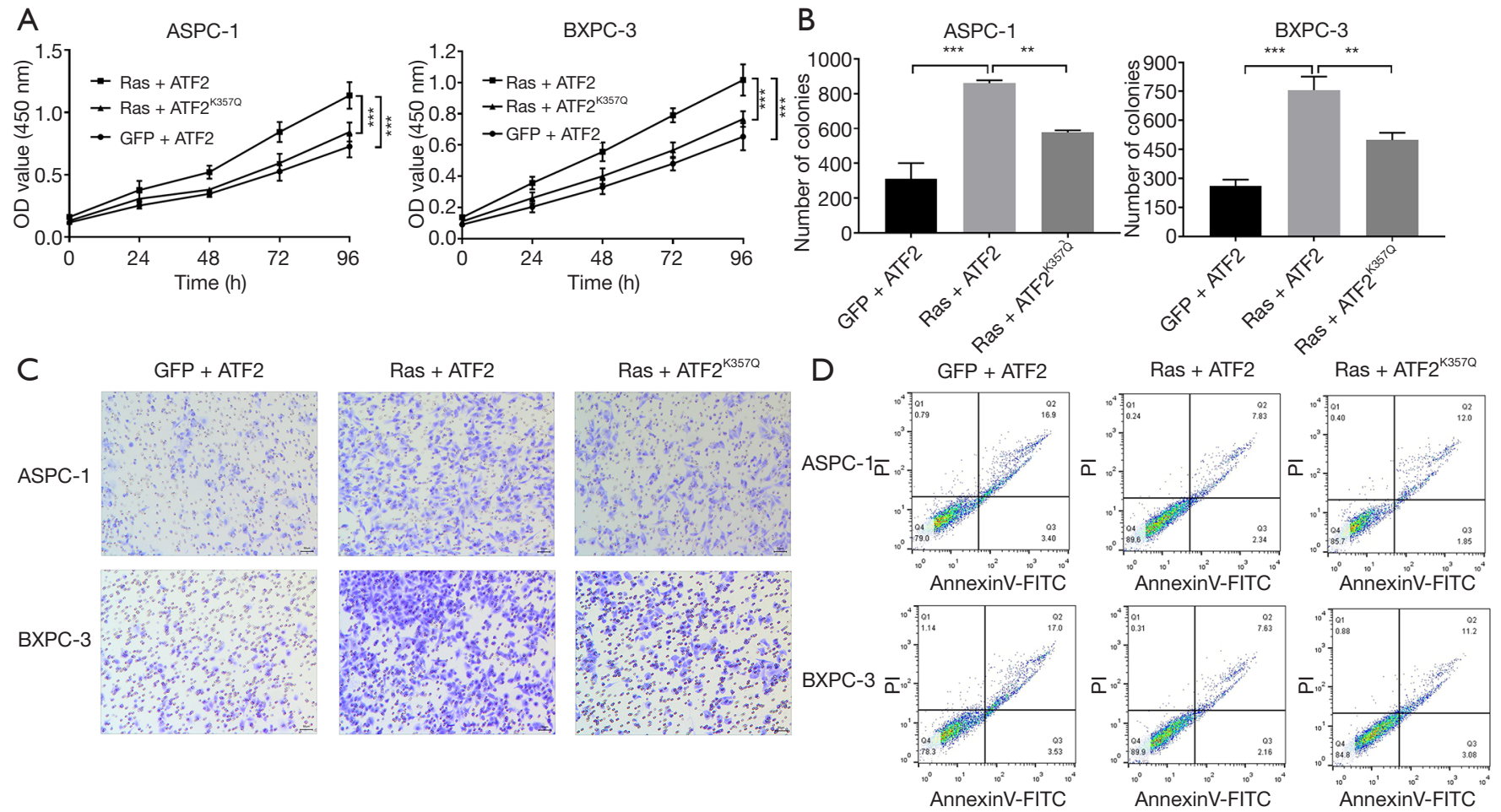

Figure $2 \mathrm{ATF}^{\mathrm{K} 357 \mathrm{ac}}$ inhibits cell proliferation and migration mediated by the Ras-ERK pathway. (A) Cell proliferation, (B) colony formation, (C) cell migration, and (D) cell apoptosis were determined using CCK8, soft agar colony formation, transwell migration (scale bar: 50 $\mu \mathrm{m})$, and flow cytometry assays, respectively. ASPC-1 and BXPC-3 cells were transfected with pEGFP-N1 (GFP), pEGFP ATF2 (ATF2), pEGFP-Ras, and ATF2 ${ }^{\mathrm{K} 357 \mathrm{Q}}$ plasmids. ${ }^{* *}, \mathrm{P}<0.01$; ${ }^{* * *}, \mathrm{P}<0.001$.

\section{Acetylation of ATF2 regulates transcription of the downstream gene of the Ras-ERK signaling pathway}

Genes (IGFBP3, WNT16B, CYR61, CARD16, GDF15, and NTSE) downstream to the Ras-ERK pathway are upregulated in cancer. To investigate the transcriptional effect of ATF2 acetylation on these genes, qRT-PCR studies were conducted. The results demonstrated that the co-transfection of $\mathrm{ATF} 2^{\mathrm{K} 357 \mathrm{Q}}$ along with Ras decreased transcription of the target genes compared to ATF2 (Figure 3A). Furthermore, in ChIP assays, we observed that ATF2 was reduced on the promoters of respective genes (Figure 3B). These results imply that ATF2 binding to the promoter region of the aforementioned genes is required for transcriptional regulation.

\section{The silencing of HDAC2 attenuates acetylated ATF2- dependent phenotypes of pancreatic cancer cells}

HDAC families of deacetylases are capable of removing acetylation modification. We aimed to explore whether
HDAC2 silencing would increase the level of acetylated ATF2. For this, two siRNAs (si-HDAC2-1 and siHDAC2-2) were synthesized to silence HDAC2, and sicon siRNA was used as a negative control. As expected, PCR results showed that the HDAC2 messenger RNA (mRNA) level was remarkably reduced only in si-HDAC2-1 or si-HDAC2-2 transfected cells but not in the case of sicon siRNA (Figure 4A). Furthermore, using western blot analysis, we examined the effect of HDAC2 silencing on the levels of acetylated ATF2 upon activation of the RasERK pathway. We found that HDAC2 silencing decreased the levels of $\mathrm{ATF} 2^{\mathrm{K} 357 \mathrm{ac}}$ in both types of pancreatic cancer cells (Figure 4B). In addition, the CCK-8, colony formation, and transwell migrations assays also demonstrated that the cell viability, proliferation, and migration enhanced by the Ras-ERK pathway were notably repressed upon silencing of HDAC2 $(\mathrm{P}<0.001)$ (Figures $4 C, D, E)$, whereas apoptosis was elevated (Figure 4F). Altogether, these results emphasize the importance of acetylated ATF2 in maintaining cancer cell phenotypes. 
A

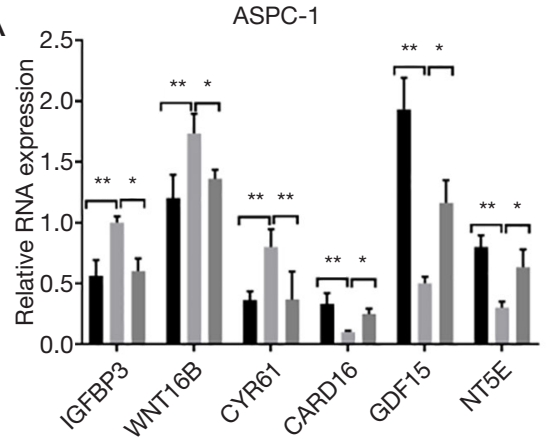

- GFP + ATF2

- Ras + ATF2

- Ras + ATF2 ${ }^{\mathrm{K} 3570}$
B

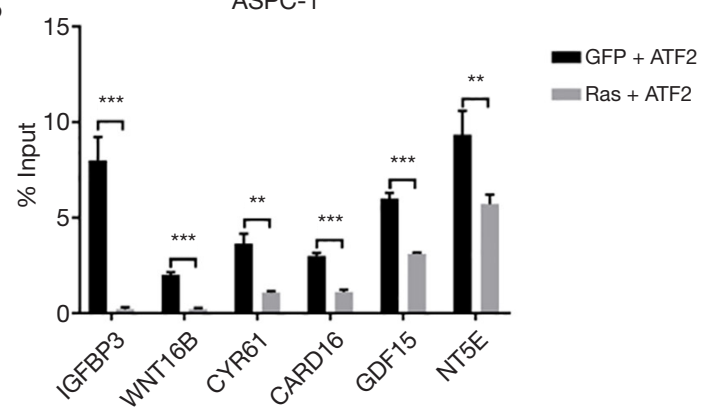

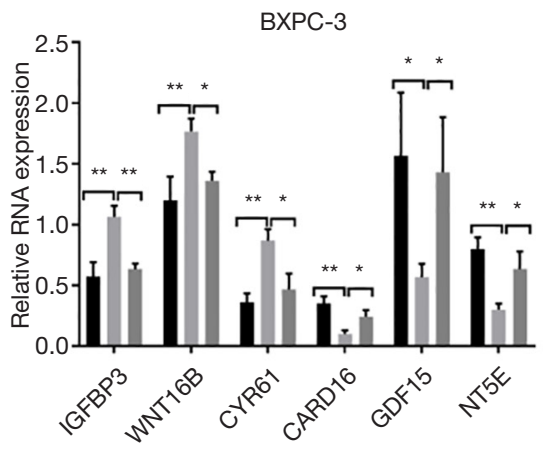

BXPC-3

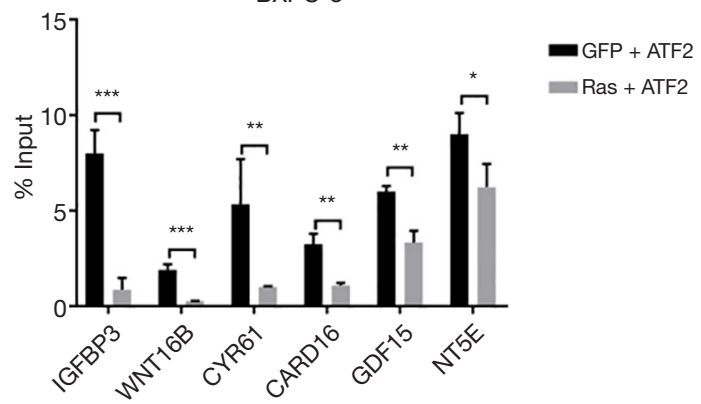

Figure $3 \mathrm{ATF}^{\mathrm{K} 357 \mathrm{ac}}$ modulates the transcription of downstream genes of the ERK1/2 pathway. (A) The mRNA expression of ERK1/2 downstream genes (WNT16B, IGFBP3, CYR61, CARD16, NT5E, and GDF15) in pEGFP-N1 (GFP), pEGFP ATF2 (ATF2), Ras ${ }^{\text {G12V/T35s }}$ (Ras), and ATF2 ${ }^{\mathrm{K} 357 \mathrm{Q}}$ transfected cells were assessed by qRT-PCR. (B) The transcription of CARD16, IGFBP3, NTSE, WNT16B, CYR61, and GDF15 genes in pEGFP ATF2 (ATF2) and Ras ${ }^{\text {G12V/T35S }}$ (Ras) transfected cells following the activation of the Ras-ERK pathway were evaluated by ChIP assay. ${ }^{*}, \mathrm{P}<0.05 ;{ }^{* *}, \mathrm{P}<0.01 ;{ }^{* * *}, \mathrm{P}<0.001$. The percent of expression was normalized to GAPDH expression.

\section{ATF2 $2^{K 37 a c}$ is downregulated by 1300 degradation, induced by the Ras-ERK pathway}

The p300 protein is a coactivator that has intrinsic acetyltransferase activity and is known to interact with ATF2. Western blot analysis was performed to investigate whether p300 was involved in regulating the levels of ATF2 ${ }^{\mathrm{K} 357 \mathrm{ac}}$. Our results showed that HDAC2 and p300 protein levels were reduced upon activation of the Ras-ERK pathway (Figures $5 A, B$ ). The reduced level of p300 downregulated the ATF2 ${ }^{\mathrm{K} 357 \mathrm{ac}}$ in the ASPC-1 and BXPC-3 cells (Figure 5C). However, we found that the mRNA level of p300 and HDAC2 was not affected (Figure 5D). Subsequently, ChIP assays revealed that $\mathrm{p} 300$ could bind to the promoter region of downstream target genes (CAFRD16, CYR61, WNT16B, IGFBP3, GDF15, and NTSE) regulated by ATF2, however was downregulated after pathway activation, along with the downregulation of ATF2 (Figure 5E).

To further confirm the involvement of p300 in regulation, MG132 proteasome inhibitor was added into ASPC-1 and BXPC-3 cells. In contrast to Figure 5B, p300 levels were not decreased (Figure $5 F$ ). Upon activation of the Ras-EKR pathway (using Ras ${ }^{\mathrm{G} 12 \mathrm{~V} / \mathrm{T} 35 \mathrm{~S}}$ in the absence of MG132), we noticed a reduction in the levels of $\mathrm{ATF} 2^{\mathrm{K} 357 \mathrm{ac}}$ at $48 \mathrm{~h}$ posttransfection (Figure $5 G$ ). However, after 3 h of MG132 addition, the level of ATF $2^{\mathrm{K} 357 \mathrm{ac}}$ recovered (Figure $5 \mathrm{H}$ ). These results indicated that the degradation of $\mathrm{p} 300$ downregulated $\mathrm{ATF} 2^{\mathrm{K} 357 \mathrm{ac}}$.

\section{p300 degradation was mediated by MDM2, induced by the Ras-ERK pathway}

E3 ubiquitin ligase MDM2 is associated with the degradation of various histone acetylates. So, we investigated the possible involvement of MDM2 in the degradation of p300 following activation of the Ras-ERK pathway. ASPC-1 and BXPC-3 cells were transfected with MDM2-His along with K-Ras ${ }^{\mathrm{G} 12 \mathrm{~V} / \mathrm{T} 35 \mathrm{~S}}$. After $48 \mathrm{~h}$ of transfection, the levels of exogenous and endogenous p300 were detected using anti-HA and anti-p300 antibodies, respectively. The results showed that Ras ${ }^{\mathrm{G} 12 \mathrm{~V} / \mathrm{T} 35 \mathrm{~S}}$ and MDM2 could significantly reduce p300 protein levels (Figures $6 A, B$ ). $\mathrm{MDM}^{\mathrm{C} 464 \mathrm{~A}}$, a 
A

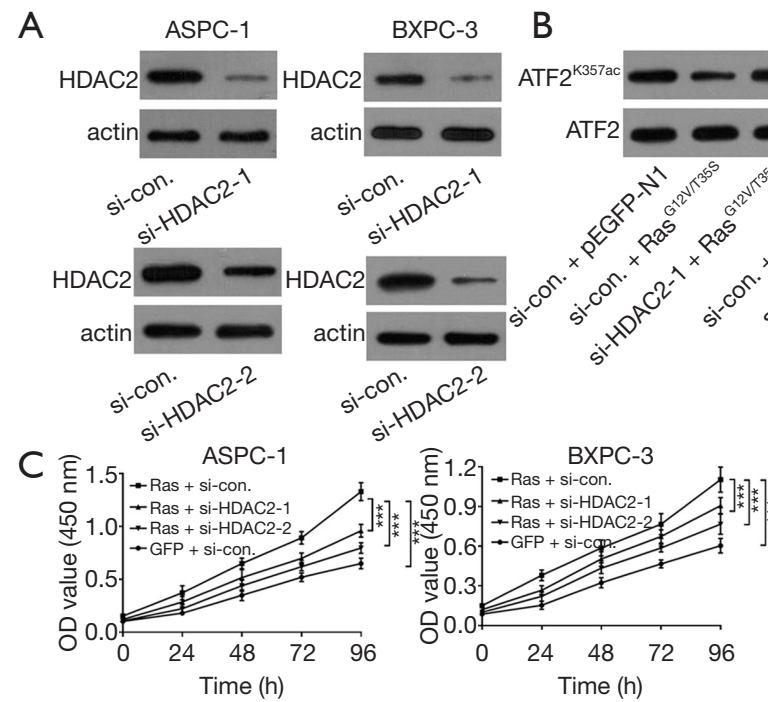

BXPC-3

B

ASPC-1

BXPC-3
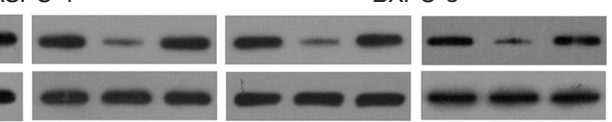

$E$

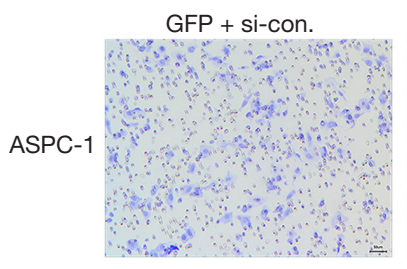

Ras + si-con.

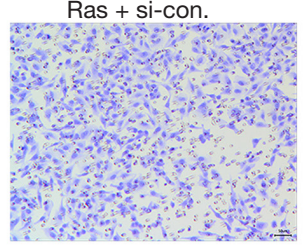

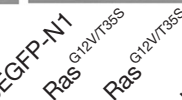

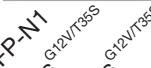

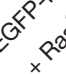

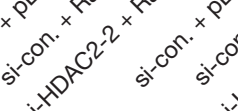

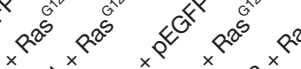

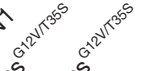

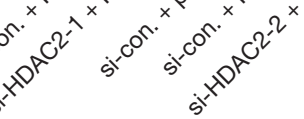

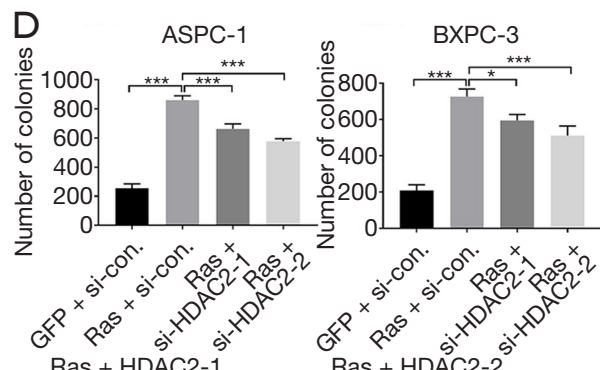

Ras + HDAC2-1

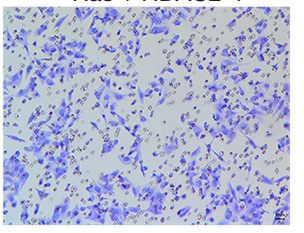

Ras + HDAC2-2
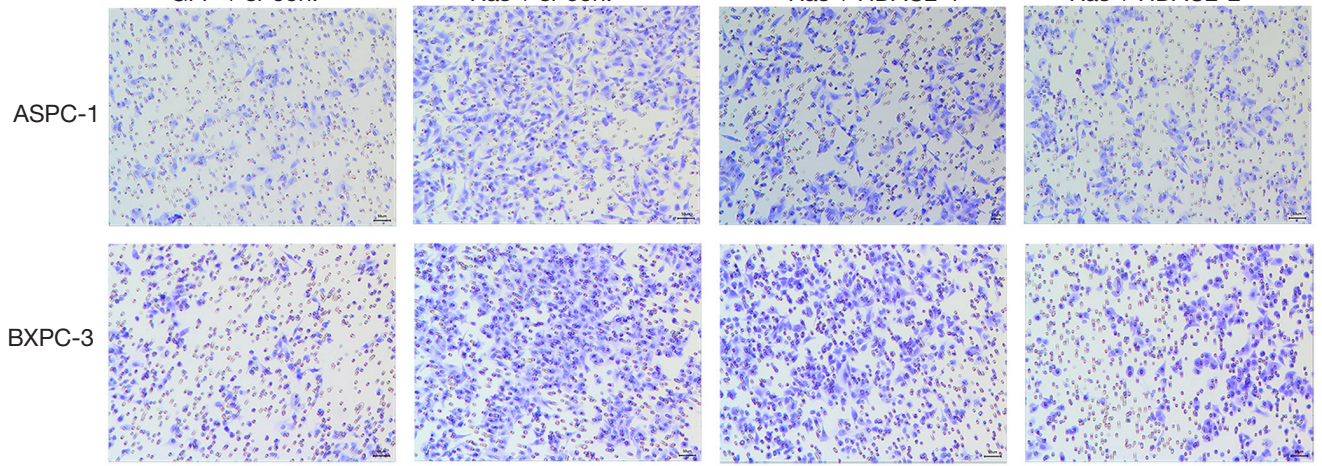

$\mathrm{F}$
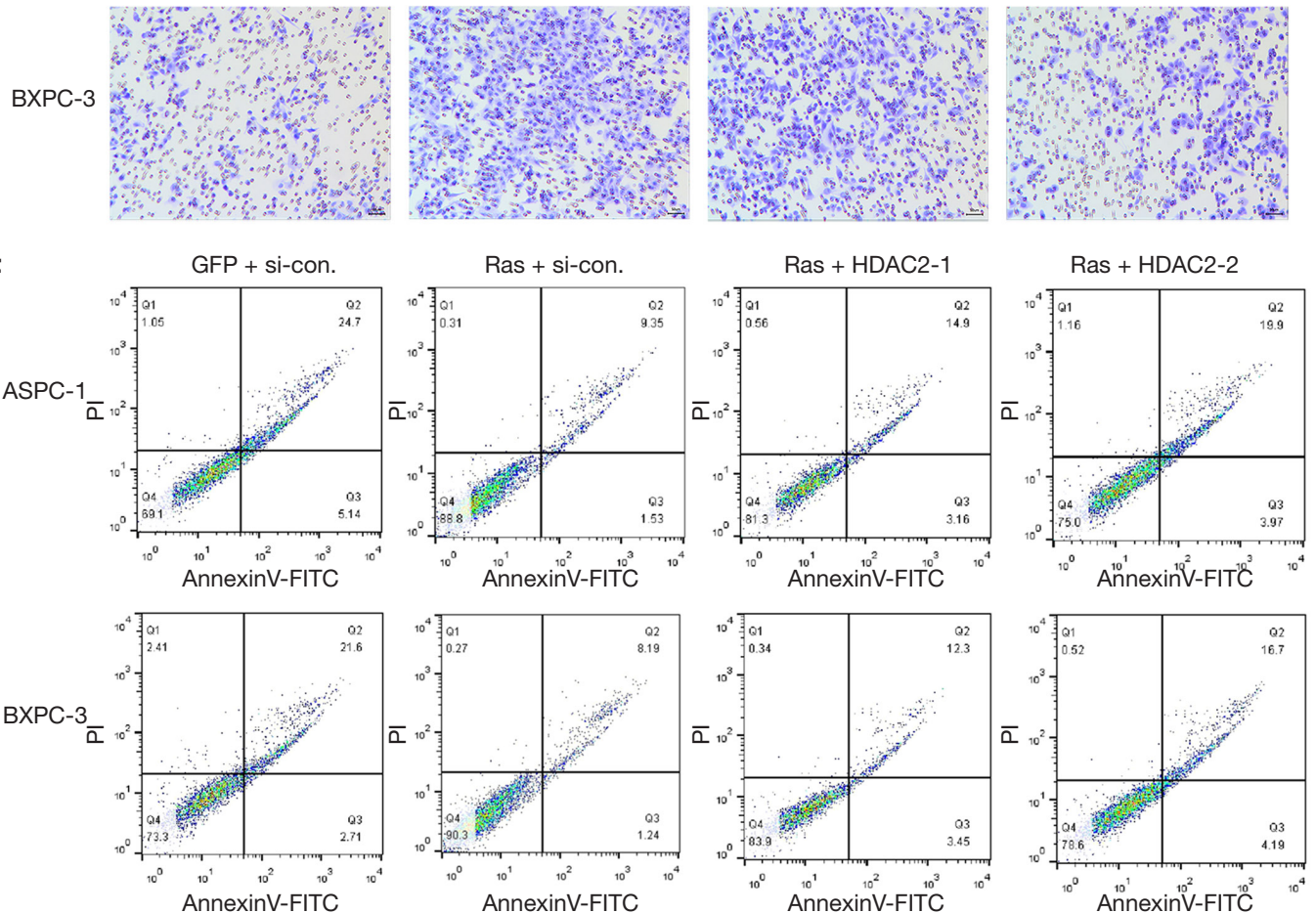

Figure 4 HDAC2 silencing attenuates the ATF2 ${ }^{\mathrm{K} 357 \mathrm{ac}}$-dependent phenotypes of pancreatic cancer cells. (A) The HDAC2 mRNA expression of ASPC-1 and BXPC-3 cells transfected with si-HDAC2-1 and si-HDAC2-2 were detected by PCR. (B) ATF2 ${ }^{\text {K357ac }}$ expression in pEGFP-N1 (GFP), Ras ${ }^{\text {G12V/T35s }}$ (Ras), si-HDAC2-1, si-HDAC2-2, and si-con transfected cells was detected by western blot. (C) CCK8 assay was utilized to assess cell proliferation of transfected cells. (D) The colony number was detected by soft agar colony formation. (E) Transwell migration assay was utilized to determine the migration of transfected cells (scale bar: $50 \mu \mathrm{m}$ ). (F) The apoptosis of transfected cells was evaluated by flow cytometry. ${ }^{* *}, \mathrm{P}<0.001$. 

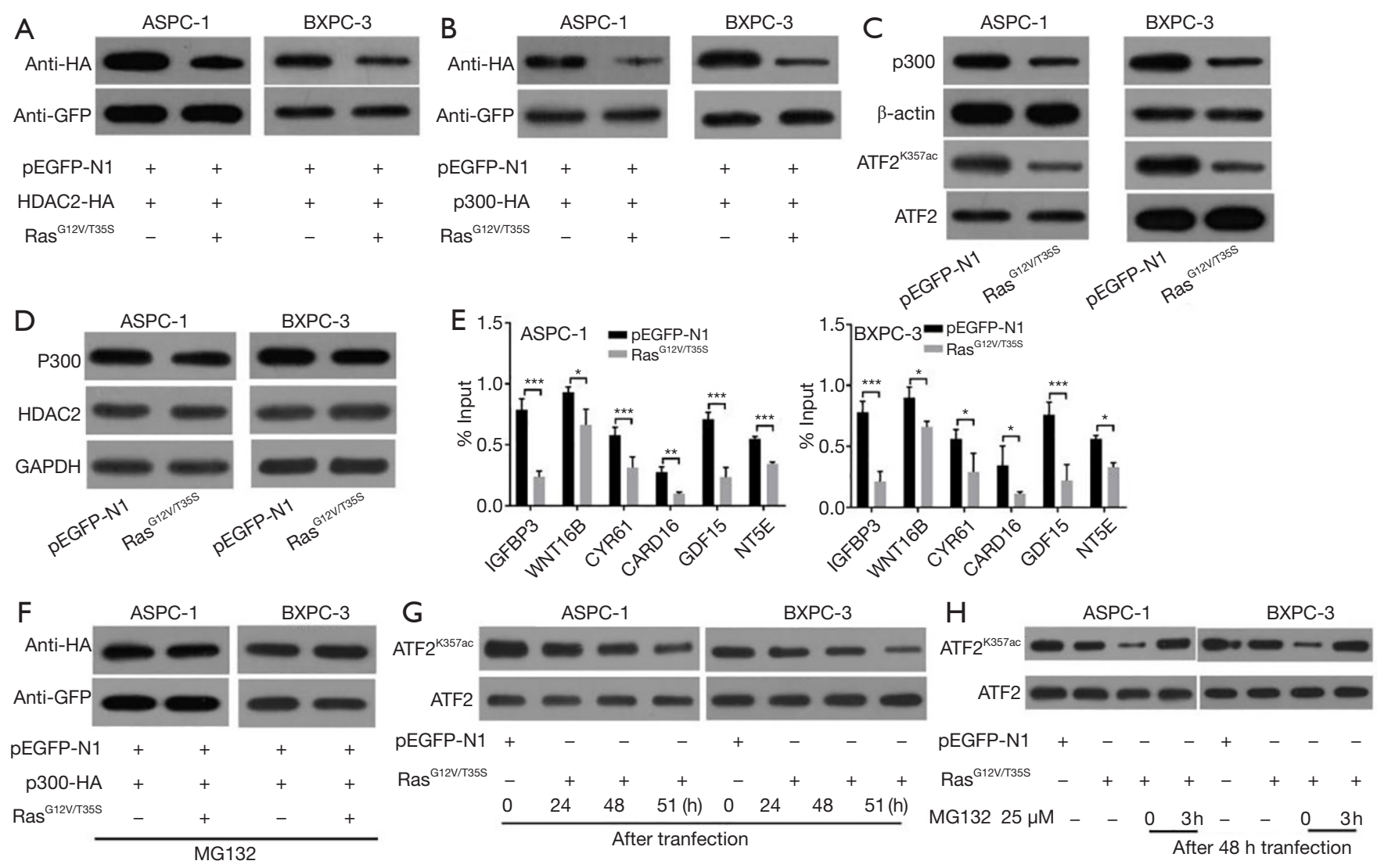

Figure 5 ATF2 ${ }^{\mathrm{K} 357 \mathrm{ac}}$ downregulation is caused by p300 degradation, induced by the activation of the Ras-ERK pathway. (A) Western blot was used to estimate the protein level in pEGFP-N1, HDAC2-HA, and Ras ${ }^{\text {G12V/T35s }}$ transfected cells. (B) Western blot was used to estimate the protein levels in pEGFP-N1, p300-HA, and Ras ${ }^{\mathrm{G} 12 \mathrm{~V} / \mathrm{T} 35 \mathrm{~S}}$ transfected cells. (C) Western blot was used to estimate the levels of p300-HA and $\mathrm{ATF}^{\mathrm{K} 357 \mathrm{ac}}$ in pEGFP-N1 and Ras ${ }^{\mathrm{G} 12 \mathrm{~V} / \mathrm{T} 35 \mathrm{~S}}$ transfected cells. (D) The mRNA expression of p300 and HDAC2 in pEGFP-N1 and Ras ${ }^{\mathrm{G} 12 \mathrm{~V} / \mathrm{T} 35 \mathrm{~S}}$ transfected cells were determined by PCR gel electrophoresis. (E) ChIP assay was used to determine IGFBP3, WNT16B, CYR61, CARD16, GDF15, and NT5E transcriptions in pEGFP-N1 and Ras ${ }^{\text {G12V/T35s }}$ transfected cells. (F) Western blot was used to estimate the levels of p300 protein in pEGFP-N1 and Ras ${ }^{\text {G12V/T35S }}$ transfected cells after MG132 protease inhibitor treatment. (G) Western blot was used to estimate the level of $\mathrm{ATF}^{\mathrm{K} 357 \mathrm{ac}}$ in pEGFP-N1 and Ras ${ }^{\mathrm{G} 12 \mathrm{~V} / \mathrm{T} 35 \mathrm{~S}}$ transfected cells (transfection time: 0, 24, 48, and $51 \mathrm{~h}$ ) without MG132. (H) Western blot was used to estimate the level of ATF2 ${ }^{\mathrm{K} 357 \mathrm{ac}}$ in pEGFP-N1 and Ras ${ }^{\mathrm{G} 12 \mathrm{~V} / \mathrm{T} 35 \mathrm{~S}}$ transfected cells with MG132 (25 $\mu$ M, treatment time: 0 and 3 h). *, $\mathrm{P}<0.05 ;{ }^{* *}, \mathrm{P}<0.01,{ }^{* * *} ; \mathrm{P}<0.001$.

really interesting new gene (RING)-finger domain mutant lacks ubiquitin activity. So, when MDM2 ${ }^{\mathrm{C} 464 \mathrm{~A}}$ (MDM2MU) was transfected, protein levels of p300 did not change significantly (Figures 6C,D), indicating that the degradation of p300 was dependent on MDM2.

Furthermore, we analyzed whether activation of the Ras-ERK pathway upregulated MDM2 to enhance p300 degradation. Results showed that activation of the Ras-ERK pathway significantly increased the level of endogenous MDM2 (Figure 6E). To examine whether the upregulation of MDM2 increased p300 degradation, the MDM2 level was decreased by using siRNA (si-MDM2) against MDM2 (Figure 6F). Interestingly, the downregulation of ATF2 ${ }^{\mathrm{K} 357 \mathrm{ac}}$ was inhibited after the transfection of si-MDM2 (Figure 6G). Overall, these results suggested that the RasERK pathway, which regulates ATF2 ${ }^{\mathrm{K} 357 \mathrm{ac}}$ induced p300 degradation through MDM2.

\section{Discussion}

A hyper-activated Ras-ERK pathway is closely associated with pancreatic cancer, particularly in the regulation of 
A

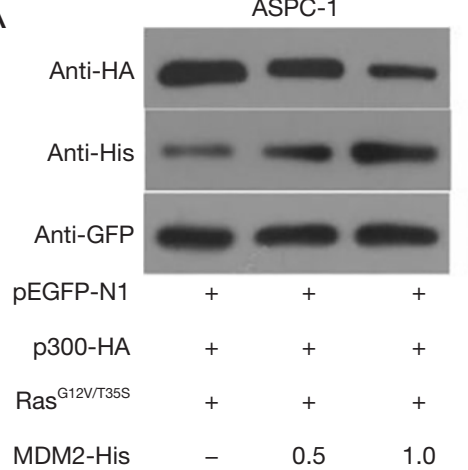

C

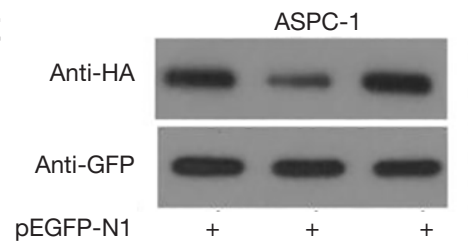

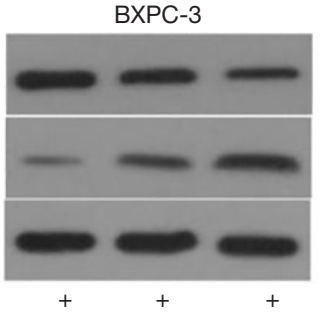

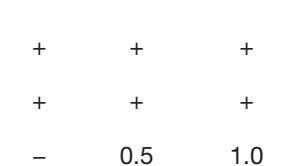

$-\quad 0.5 \quad 1.0$

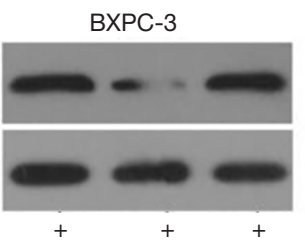

B

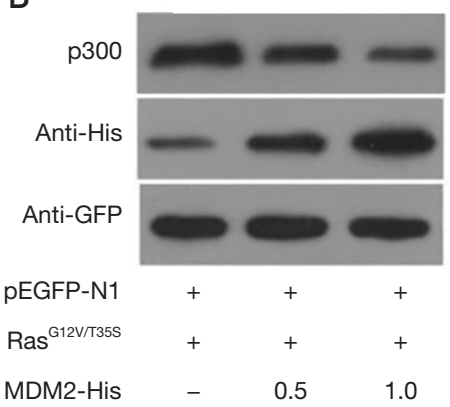

MDM2-His

D
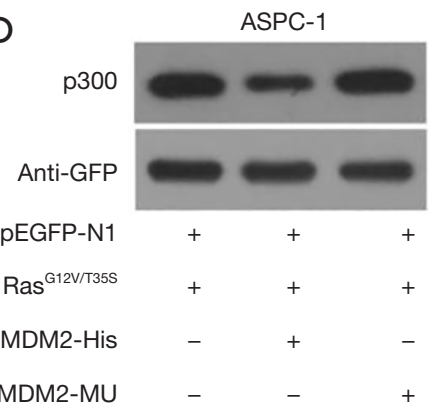

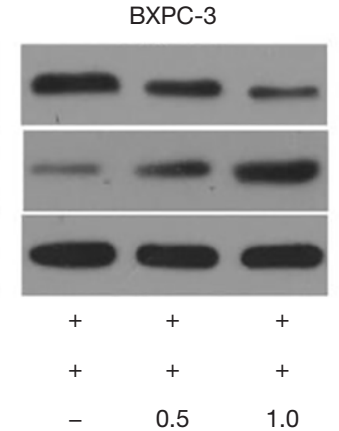

BXPC-3

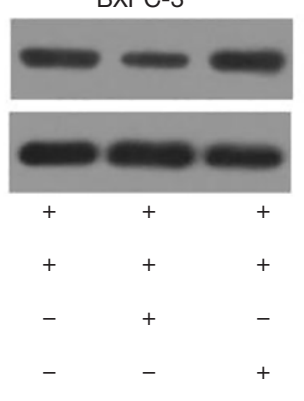

$\mathrm{E}$

MDM2-MU
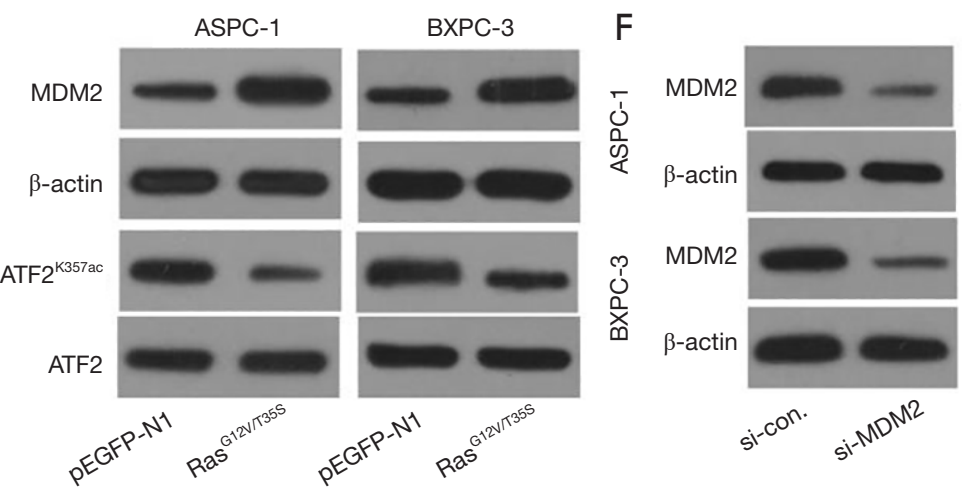

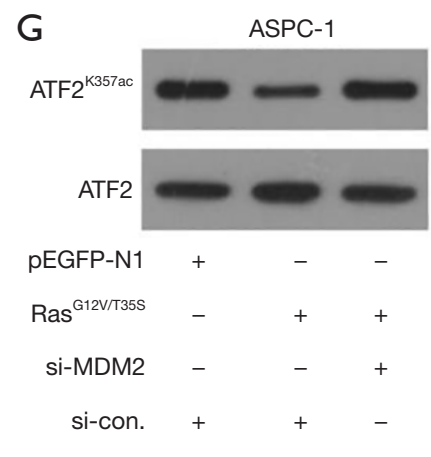

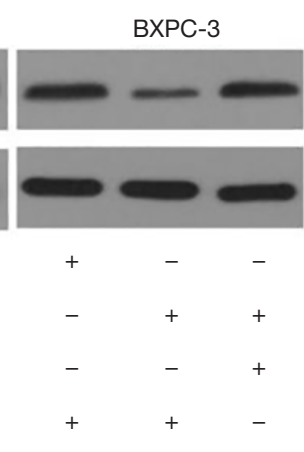

Figure 6 p300 degradation is mediated by MDM2, induced by the activated Ras-ERK pathway. The exogenous and (B) endogenous p300 levels in cells transfected with pEGFP-N1, p300-HA, Ras ${ }^{\mathrm{G} 12 \mathrm{~V} / \mathrm{T} 35 \mathrm{~S}}$, and MDM2-His (0.5 and $\left.1.0 \mu \mathrm{g}\right)$. (C) The exogenous and (D) endogenous p300 levels in cells transfected with pEGFP-N1, p300-HA, Ras ${ }^{\mathrm{G} 12 \mathrm{~V} / \mathrm{T} 35 \mathrm{~S}}$, and MDM2-His in MDM2-MU transfected cells were evaluated by western blot. (E) Western blot was used to assess MDM2 and ATF2 ${ }^{\text {K357ac }}$ proteins in pEGFP-N1 and Ras ${ }^{\mathrm{G} 12 \mathrm{~V} / \mathrm{T} 35 \mathrm{~S}}$ transfected cells. (F) Western blot was used to assess MDM2 levels in cells transfected with si-MDM2 and si-con. (G) The level of ATF2 ${ }^{\mathrm{K} 357 \mathrm{ac}}$ in cells transfected with pEGFP-N1, Ras ${ }^{\mathrm{G} 12 \mathrm{~V} / \mathrm{T} 35 \mathrm{~S}}$, si-MDM2, and si-NC was assessed by Western blotting.

cell growth and migration $(9,26)$. In this study, Ras was found to be an important upstream signaling molecule that upregulates certain genes downstream of the RasERK pathway. These genes, including CYR61, WNT16B, IGFBP3, GDF15, NTSE, and CARD16, are regulators of cancer cell proliferation and migration activity (27).

$\mathrm{ATF} 2$ is also an epigenetic regulator that possesses intrinsic acetyltransferase activity. Specifically, it acts to acetylate the histones H2B and H4. The Ras-ERK pathwaymediated phosphorylation of ATF2 not only triggers its DNA-binding effectiveness but also augments its intrinsic acetyltransferase activity (21). The regulatory effect of ATF2 on histone acetylation can be extended to many amino acid-regulated genes (28). Interestingly, ATF2 can also be 
acetylated by another histone acetyltransferase, p300, at the two lysine acetylation sites, K357 and K374. These lysine residues are located in the basic leucine zipper (b-ZIP) domain of ATF2. The modification at this site(s) triggers conformational changes to alter the DNA-binding capacity of ATF2. Importantly, mutations of the b-ZIP acetylation site hinder transcriptional activation of ATF2 (25).

Numerous cancer studies have found that ATF2 is an important regulator in pancreatic cancer. However, the specific role of $\mathrm{ATF} 2^{\mathrm{K} 357 \mathrm{ac}}$ is unclear. In this study, we demonstrated that the $\mathrm{ATF} 2^{\mathrm{K} 357 \mathrm{ac}}$ regulates the characteristic phenotype of pancreatic cancer cells via the Ras-ERK pathway by regulating the expression of downstream proliferation, migration, and other related genes. We found that the level of ATF2 ${ }^{\mathrm{K} 357 \mathrm{ac}}$ is strongly associated with the development of pancreatic cancer.

It is reported that HDAC2, a deacetylase, can reverseregulate ATF2 (25). Hence, we investigated the effect of HDAC2 on the proliferation and migration of pancreatic cells using siRNA experiments, and found that the cell phenotypes are related to the expression level of HDAC2, and HDAC2 regulates ATF2 ${ }^{\mathrm{K} 357 \mathrm{ac}}$ levels. Furthermore, the activated Ras-ERK pathway induces the degradation of p300 mediated by the MDM2-dependent proteasome pathway, which in turn regulates the level of $\mathrm{ATF}^{\mathrm{K} 357 \mathrm{ac}}$.

\section{Conclusions}

However, there are some limitations to this regulatory model of ATF2 due to the presence of other enzyme molecules, especially deacetylases. These may also simultaneously participate in regulating $\mathrm{ATF} 2^{\mathrm{K} 357 \mathrm{ac}}$ by altering the acetyl modification site. Moreover, the affinity of p300 and HDAC2 to ATF2 is unknown, especially in the Ras-ERK signaling pathway-mediated regulation. The differential affinities of p300 or HDAC2 for ATF2 could be significant. It is also necessary to investigate whether the localization of $\mathrm{p} 300$ is altered, as it could have downregulated $\mathrm{ATF} 2^{\mathrm{K} 357 \mathrm{ac}}$ due to the decreased availability of p300, thereby p300 could change the affinity for ATF2. Thus, these aspects need to be explored further to comprehensively understand the regulation of $\mathrm{ATF} 2{ }^{\mathrm{K} 357 \mathrm{ac}}$.

\section{Acknowledgments}

Funding: This study was supported by the Natural Science Foundation of Liaoning Province [No. 180530068], the Liaoning Province Project of Hundred, Thousand, Ten
Thousand Talent Projects [3200417003], and the Project of Shenyang Municipal Science and Technology Bureau [17231178].

\section{Footnote}

Reporting Checklist: The authors have completed the MDAR reporting checklist. Available at http://dx.doi.org/10.21037/ atm-20-5880

Data Sharing Statement: Available at http://dx.doi. org/10.21037/atm-20-5880

Conflicts of Interest: All authors have completed the ICMJE uniform disclosure form (available at http://dx.doi. org/10.21037/atm-20-5880). The authors have no conflicts of interest to declare.

Ethical Statement: The authors are accountable for all aspects of the work in ensuring that questions related to the accuracy or integrity of any part of the work are appropriately investigated and resolved.

Open Access Statement: This is an Open Access article distributed in accordance with the Creative Commons Attribution-NonCommercial-NoDerivs 4.0 International License (CC BY-NC-ND 4.0), which permits the noncommercial replication and distribution of the article with the strict proviso that no changes or edits are made and the original work is properly cited (including links to both the formal publication through the relevant DOI and the license). See: https://creativecommons.org/licenses/by-nc-nd/4.0/.

\section{References}

1. Shi J, Xue J. Inflammation and development of pancreatic ductal adenocarcinoma. Chin Clin Oncol 2019;8:19.

2. Haeberle L, Esposito I. Pathology of pancreatic cancer. Transl Gastroenterol Hepatol 2019;4:50.

3. Feng J, Rao $M$, Wang $M$, et al. Triptolide suppresses pancreatic cancer cell proliferation by inhibiting hedgehog signaling pathway activity. Sci China Life Sci 2019;62:1409-12.

4. Ducreux M, Cuhna AS, Caramella C, et al. Cancer of the pancreas: ESMO Clinical Practice Guidelines for diagnosis, treatment and follow-up. Annals of oncology: official journal of the European Society for Medical Oncology. 2015;26 Suppl 5:v56-68. 
5. Pointer DT Jr, Al-Qurayshi Z, Hamner JB, et al. Factors leading to pancreatic resection in patients with pancreatic cancer: a national perspective. Gland Surg 2018;7:207-15

6. Sinn M, Bahra M, Liersch T, et al. CONKO-005: Adjuvant Chemotherapy With Gemcitabine Plus Erlotinib Versus Gemcitabine Alone in Patients After R0 Resection of Pancreatic Cancer: A Multicenter Randomized Phase III Trial. J Clin Oncol 2017;35:3330-7.

7. Conroy T, Ducreux M. Adjuvant treatment of pancreatic cancer. Curr Opin Oncol 2019;31:346-53.

8. O'Reilly EM, Abou-Alfa GK. Cytotoxic therapy for advanced pancreatic adenocarcinoma. Semin Oncol 2007;34:347-53.

9. Zheng $\mathrm{C}$, Jiao $\mathrm{X}$, Jiang $\mathrm{Y}$, et al. ERK1/2 activity contributes to gemcitabine resistance in pancreatic cancer cells. J Int Med Res 2013;41:300-6.

10. Kohno M, Pouyssegur J. Targeting the ERK signaling pathway in cancer therapy. Ann Med 2006;38:200-11.

11. Chadha KS, Khoury T, Yu J, et al. Activated Akt and Erk expression and survival after surgery in pancreatic carcinoma. Ann Surg Oncol 2006;13:933-9.

12. Cao AL, Tang QF, Zhou WC, et al. Ras/ERK signaling pathway is involved in curcumin-induced cell cycle arrest and apoptosis in human gastric carcinoma AGS cells. J Asian Nat Prod Res 2015;17:56-63.

13. Dorard C, Vucak G, Baccarini M. Deciphering the RAS/ ERK pathway in vivo. Biochem Soc Trans 2017;45:27-36.

14. Santos E, Crespo P. The RAS-ERK pathway: A route for couples. Sci Signal 2018;11:eaav0917.

15. Neuzillet C, Tijeras-Raballand A, de Mestier L, et al. MEK in cancer and cancer therapy. Pharmacol Ther 2014;141:160-71.

16. Neuzillet C, Hammel P, Tijeras-Raballand A, et al. Targeting the Ras-ERK pathway in pancreatic adenocarcinoma. Cancer Metastasis Rev 2013;32:147-62.

17. Wu DS, Chen C, Wu ZJ, et al. ATF2 predicts poor prognosis and promotes malignant phenotypes in renal cell carcinoma. J Exp Clin Cancer Res 2016;35:108.

18. Zhang R, Luo H, Wang S, et al. MiR-622 suppresses proliferation, invasion and migration by directly targeting activating transcription factor 2 in glioma cells. J Neurooncol 2015;121:63-72.

19. Lo Iacono M, Monica V, Vavalà T, et al. ATF2 contributes to cisplatin resistance in non-small cell lung cancer and celastrol induces cisplatin resensitization through inhibition of JNK/ATF2 pathway. Int J Cancer 2015;136:2598-609.

20. Zhang S, Dong X, Ji T, et al. Long non-coding RNA UCA1 promotes cell progression by acting as a competing endogenous RNA of ATF2 in prostate cancer. Am J Transl Res 2017;9:366-75.

21. Kawasaki H, Schiltz L, Chiu R, et al. ATF-2 has intrinsic histone acetyltransferase activity which is modulated by phosphorylation. Nature 2000;405:195-200.

22. Vlahopoulos SA, Logotheti S, Mikas D, et al. The role of ATF-2 in oncogenesis. Bioessays 2008;30:314-27.

23. Janknecht R, Hunter T. Transcription. A growing coactivator network. Nature 1996;383:22-3.

24. Ogryzko VV, Schiltz RL, Russanova V, et al. The transcriptional coactivators $\mathrm{p} 300$ and CBP are histone acetyltransferases. Cell 1996;87:953-9.

25. Karanam B, Wang L, Wang D, et al. Multiple roles for acetylation in the interaction of p300 HAT with ATF-2. Biochemistry 2007;46:8207-16.

26. Liu Y, Wang DL, Chen S, et al. Oncogene Ras/ phosphatidylinositol 3-kinase signaling targets histone $\mathrm{H} 3$ acetylation at lysine 56. J Biol Chem 2012;287:41469-80.

27. Li Y, Sun W, Sun D, et al. Ras-ERK1/2 signaling promotes the development of uveal melanoma by downregulating H3K14ac. J Cell Physiol. 2019. [Epub ahead of print].

28. Bruhat A, Chérasse Y, Maurin AC, et al. ATF2 is required for amino acid-regulated transcription by orchestrating specific histone acetylation. Nucleic Acids Res 2007;35:1312-21.
Cite this article as: Li M, Song SW, Ge Y, Jin JY, Li XY, Tan XD. The Ras-ERK signaling pathway regulates acetylated activating transcription factor 2 via p300 in pancreatic cancer cells. Ann Transl Med 2020;8(19):1234. doi: 10.21037/atm-20-5880 
Supplementary

Table S1 Representative gene primers

\begin{tabular}{ll}
\hline Gene & Primer sequences (5'-3') \\
\hline IGFBP3 & Forward: 5'-CGCTACAAAGTTGACTACGAGTC-3' \\
& Reverse: 5'-GTCTTCCATTCTCTACGGCAGG-3' \\
WNT16B & Forward: 5'-TGCTCGTGCTGTTCCCCTAC-3' \\
& Reverse: 5'-ATCATGCAGTTCCATCTCTC-3' \\
CYR61 & Forward: 5'-TCCAGCCCAACTGTAAACATCA-3' \\
& Reverse: 5'-GGACACAGAGGAATGCAGCC-3' \\
CARD16 & Forward: 5'-GCCATGGCCGACAAGGT-3' \\
& Reverse: 5'-ACCTAGGAAGGAAGTACTATTTAG-3' \\
GDF15 & Forward: 5'-GACCCTCAGAGTTGCACTCC-3' \\
& Reverse: 5'-GCCTGGTTAGCAGGTCCTC-3' \\
NT5E & Forward: 5'-GTATTAGGGTATTATTTGGTTTAT-3' \\
& Reverse: 5'-CTTACCACACTCTACCATCC-3' \\
GAPDH & Forward: 5'-GTCGCCAGCCGAGCC-3' \\
& Reverse: 5'-CCCAATACGACCAAATCCGT-3'
\end{tabular}

